\title{
Smart macro-porous salecan/poly(N,N-diethylacrylamide) semi-IPN hydrogel for anti-inflammatory drug delivery
}

Wei Wei, Xiaoliang Qi, Gancheng Zuo, Junjian Li, Wei Sheng, Jianfa Zhang, Wei Dong*

Center for Molecular Metabolism, Nanjing University of Science \& Technology, Nanjing 210094, China

*Corresponding author. Fax: +86-25-84318533. Telephone: +86-25-84318533.

E-mail: weidong@njust.edu.cn 
Table S1 Weight percentage of hydrogel samples

\begin{tabular}{|c|c|c|}
\hline Sample codes & Salecan(\%) & DEA(\%) \\
\hline PDE & 0 & 100 \\
\hline SPD1 & 9.77 & 90.23 \\
\hline SPD2 & 12.62 & 87.38 \\
\hline SPD3 & 15.29 & 84.71 \\
\hline SPD4 & 17.80 & 82.20 \\
\hline
\end{tabular}

The weight of BIS and APS were $1.4 \% \mathrm{~g} / \mathrm{g}$ of DEA.

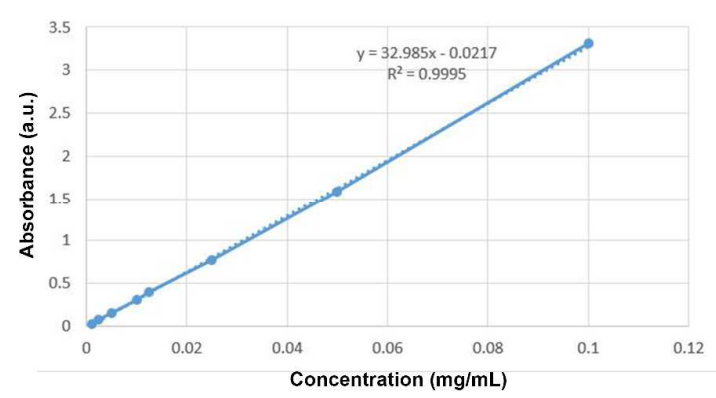

Figure S1 Standard calibration curve of Diclofenac sodium (DS) solution. (Calculate by Microsoft Office Excel 2016). 


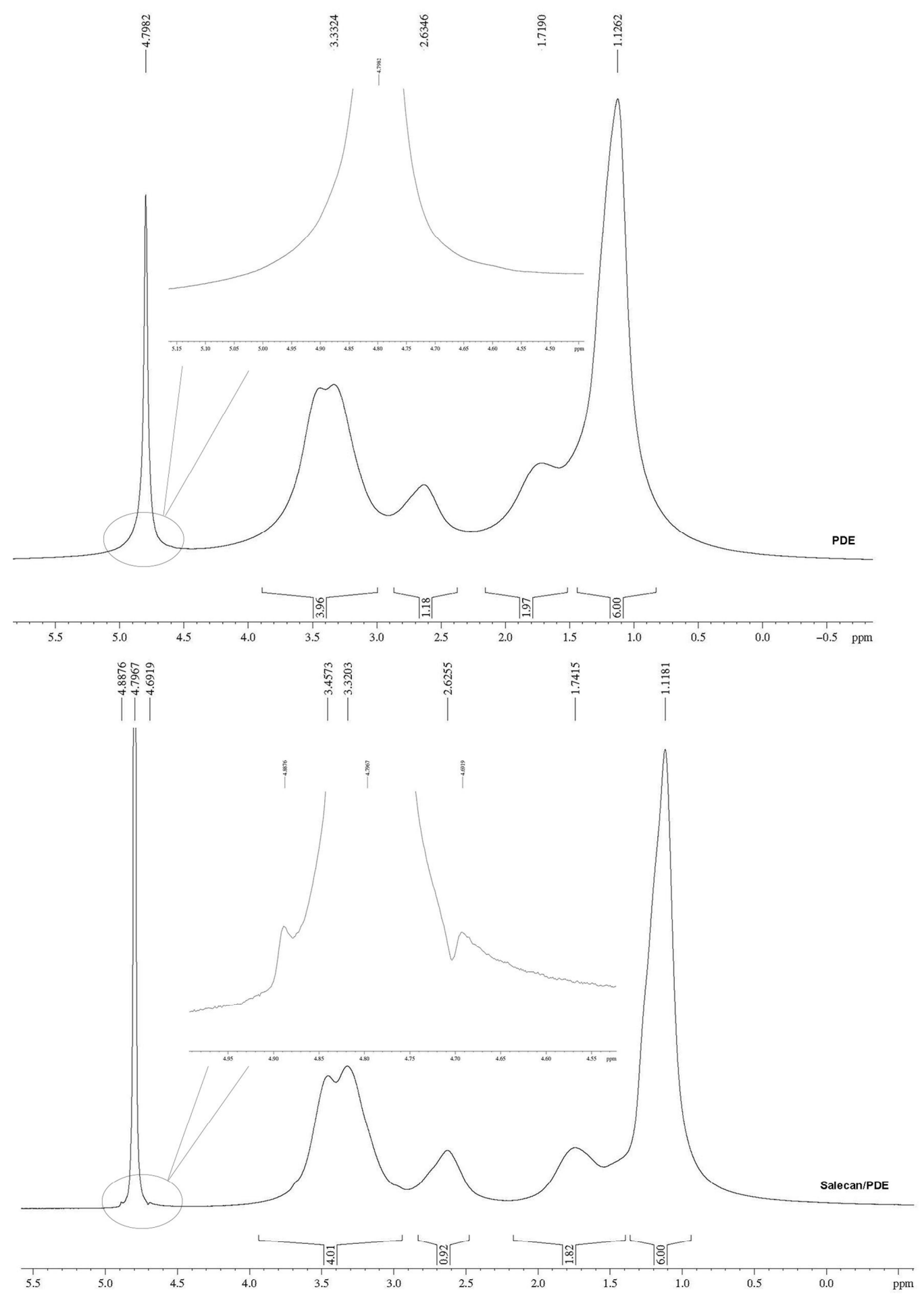

Figure S2 ${ }^{1} \mathrm{H}-\mathrm{NMR}$ spectra of salecan/PDE and pure PDE hydrogel. 


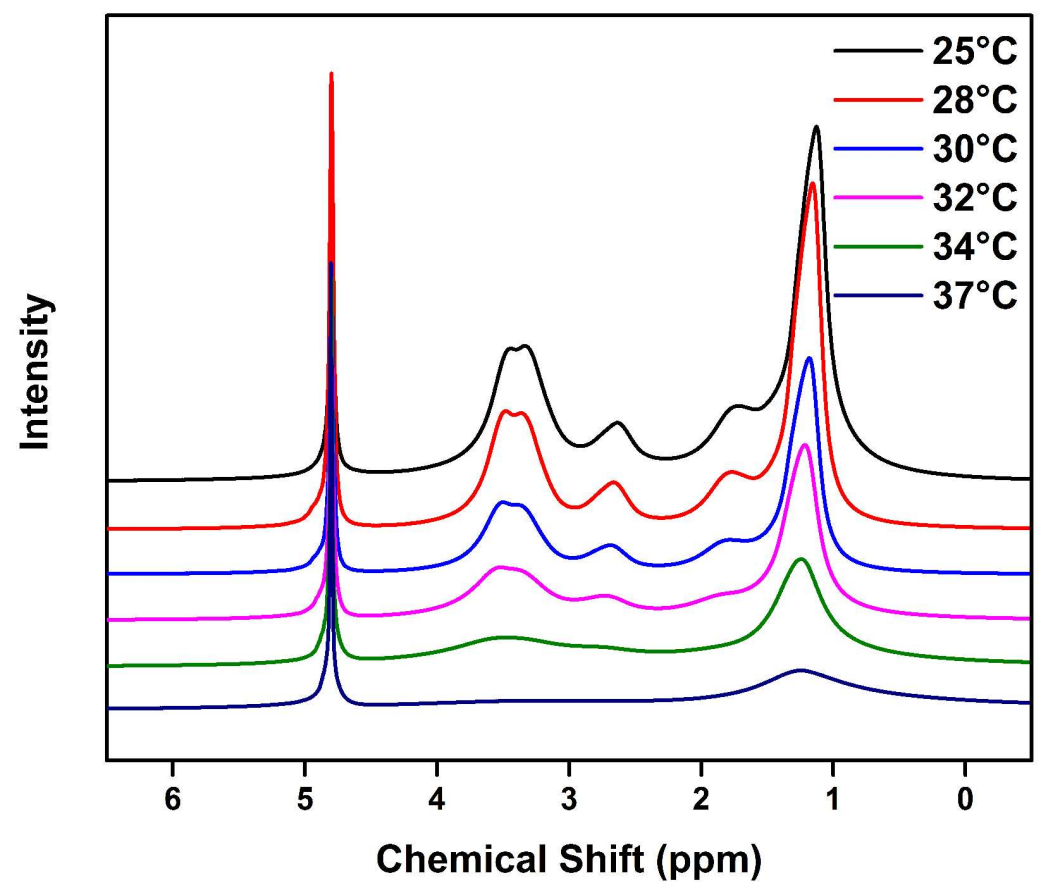

Figure S3 1H-NMR spectra of PDE hydrogel at different temperatures. 


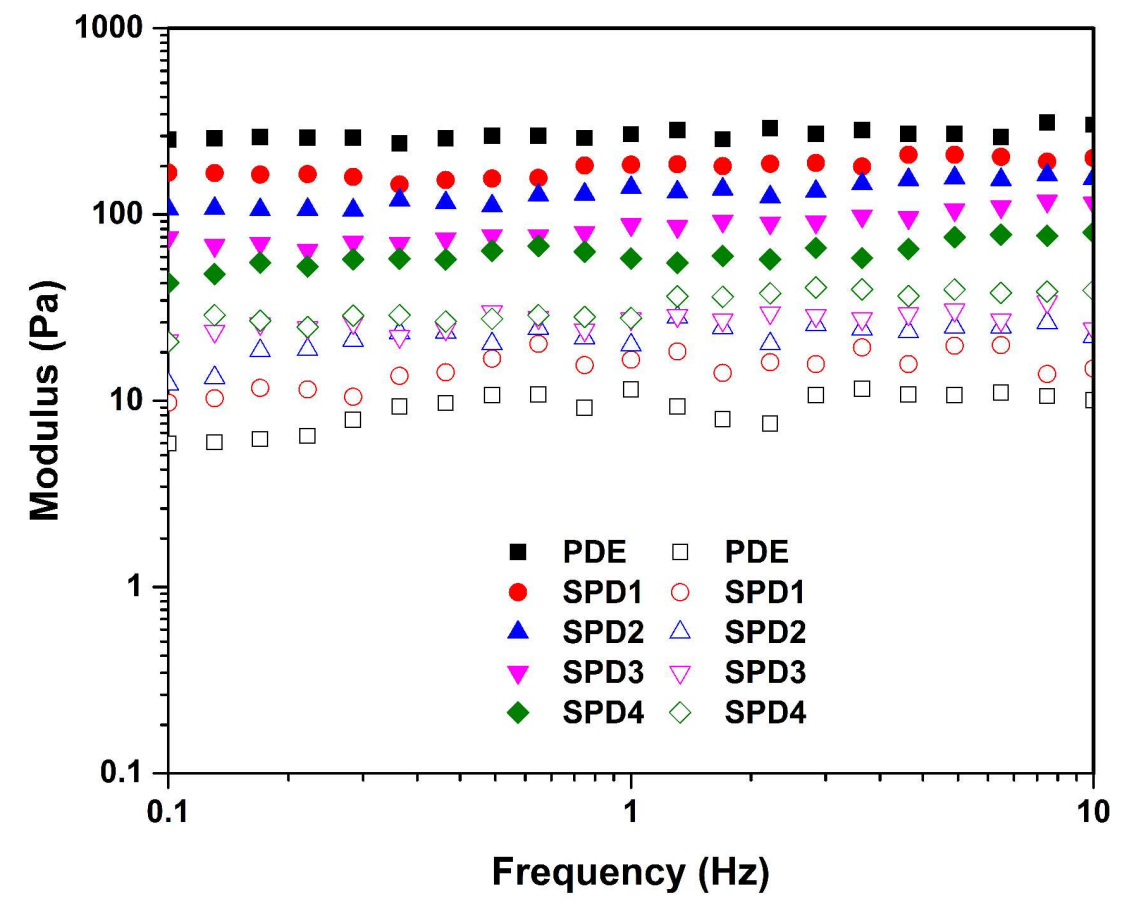

Figure S4 The storage modulus G'(closed symbols) and loss modulus G' (open symbols) of hydrogels as a function of frequency at $25^{\circ} \mathrm{C}$.

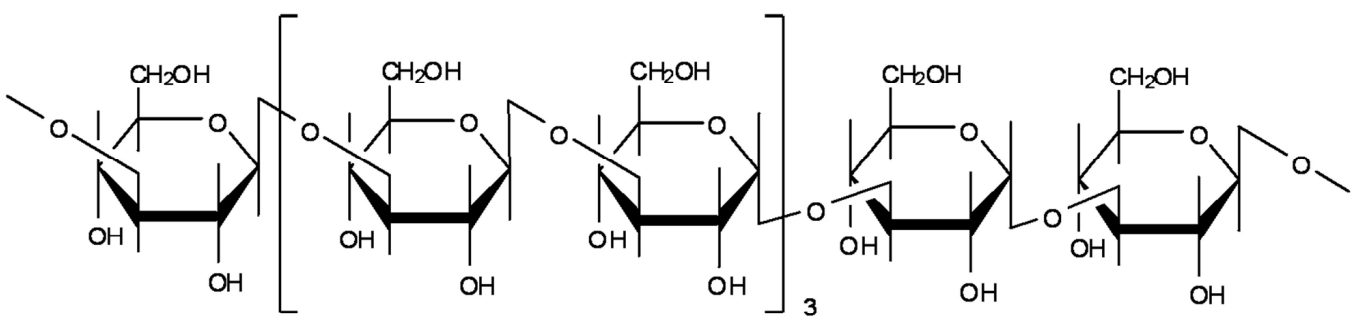

Figure S5 The structure of salecan. 


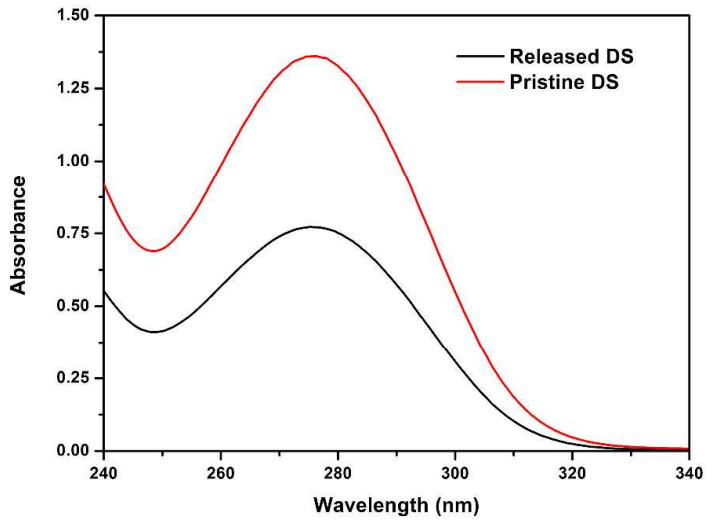

Figure S6 UV-vis absorption spectra of pristine and released DS. 